\title{
GLOBAL SINGULAR BOUNDARY METHOD FOR SOLVING 2D NAVIER-STOKES EQUATIONS
}

\author{
JURAJ MUŽÍK \& ROMAN BULKO \\ Department of Geotechnics, Faculty of Civil Engineering, University of Zilina, Slovakia
}

\begin{abstract}
This article presents a numerical algorithm based on the singular boundary method (SBM) for the incompressible steady Navier-Stokes equations formulated using primitive variables. The SBM with the Stokeslet fundamental solution and dual reciprocity (DR) principle has been chosen to solve the nonlinear flow equations. The particular solution of the non-homogeneous Stokes equations is constructed as a linear combination of implicitly local radial basis function. The simple direct iterative scheme was used to handle nonlinearities of Navier-Stokes equations with a variation of the non-homogeneous term of the particular solution. The non-homogeneous term is formed using the nonlinear convective terms of the momentum equations, evaluated using values from previous iterations. It is found that SBM with a localized DR principle gives reasonable results for numerical problems of lid-driven cavity flow up to $\operatorname{Re}=3200$, and the backward-facing step at $\operatorname{Re}=800$.

Keywords: singular boundary method, dual reciprocity, Navier-Stokes equation, Stokeslet.
\end{abstract}

\section{INTRODUCTION}

The solution of Navier-Stokes (NS) equations is one of the basic tasks of computational hydromechanics. Together with the continuity equation, it is a system of nonlinear equations. This system has already been clarified by a number of numerical methods, starting with the finite difference method through finite element method to modern meshless methods. The meshless methods consist of the smooth particle hydrodynamics (SPH) [1], the least square collocation meshless method [2]-[4], the meshless local Petrov-Galerkin (MLPG) method [5], [6], the local boundary integral element method (LBIEM) [7]-[9], and the radial basis integral equation method (RBIEM) [10]-[12]. All of these methods have advantages as well as shortcomings. One category of numerical methods used to solve the NS equations is a group of the formulas based on the boundary integral, such as e.g. boundary element method (BEM). The methods based on the boundary integral theory are represented by the BEM [1], [2], the method of fundamental solutions (MFS) [3], [4], and the singular boundary method. In the case of BEM, the singularities of the fundamental solution of the solved problem are handled by proper integration method, the MFS overcomes the singularity using a fictitious boundary, but the optimum location of this boundary remains the open problem especially for complex-shaped domains. To bypass the fictitious boundary construction, the singular boundary method (SBM) formulation adopts a concept of the origin intensity factors (OIFs). Several techniques have been developed to determine the source intensity factors, namely, inverse interpolation technique (IIT), subtracting and adding-back regularization and empirical formulas [8], [9]. In this article, the regularized boundary integral equation is used to derive a formula for Dirichlet origin intensity factors.

For the problems governed by the NS equation, these methods have to use a connection with complementary methods to be able to effectively handle nonlinear transport members of this system. The final solution is represented as a combination of the singular boundary integral and particular solutions. In this work, we present the possibility how to solve the steady fluid flow governed by the primitive variable form of NS equations using SBM with dual reciprocity (DR) scheme used to tackle the in-homogeneities brought by nonlinear transport terms of NS equations. Section 2 of our paper introduces NS equations in primitive 
variable form. Section 3 contains a brief description of the SBM and the connection with the DRM. Section 4 is dedicated to the two numerical examples and the last section, Section 5, contains the conclusions.

\section{GOVERNING EQUATIONS}

Incompressible steady flow is governed by Navier-Stokes equations in the closed domain $\Omega$ with the boundary $\Gamma$, which can be written in their primitive variables as

$$
\begin{gathered}
\frac{\partial u_{i}}{\partial x_{i}}=0, \\
\mu \frac{\partial^{2} u_{i}}{\partial x_{j} \partial x_{j}}-\frac{\partial p}{\partial x_{i}}=\rho \frac{\partial}{\partial x_{j}}\left(u_{j} u_{i}\right)+f_{i},
\end{gathered}
$$

where $u_{i}$ is the velocity vector component in the direction $i, p$ is the pressure, $v$ is the kinematic viscosity, $\rho$ is the density of a liquid, and $f_{i}$ represents body forces component in the direction $i$. The flow should satisfy boundary conditions in the form

$$
\begin{gathered}
u_{i}\left(x_{D}\right)=f_{i}\left(x_{D}\right) x_{D} \in \Gamma_{D}, \\
t_{i}\left(x_{N}\right)=\sigma_{i j} n_{j}\left(x_{N}\right) x_{N} \in \Gamma,
\end{gathered}
$$

where $u_{i}$ are the velocities and $\underline{t}_{i}$ are the tractions on the boundary and $n_{j}$ represents the outward normal to the boundary. The stress tensor is defined as

$$
\sigma_{i j}=-p \delta_{i j}+\mu\left(\frac{\partial u_{i}}{\partial x_{j}}+\frac{\partial u_{j}}{\partial x_{i}}\right) .
$$

The solution of eqns (1) and (2) can be defined as eqn (6):

$$
u_{i}=u_{i}^{\mathrm{H}}+u_{i}^{\mathrm{P}}, p=p^{\mathrm{H}}+p^{\mathrm{P}},
$$

where $u^{\mathrm{H}}$ are the solutions of the homogeneous problem that captures the Stokes equations together with the boundary conditions (eqns (3) and (4)) and $u^{\mathrm{P}}$ and $p^{\mathrm{P}}$ are the particular solutions of the non-homogeneous eqns (1) and (2). The homogeneous problem can be determined using the SBM and the particular solution is formulated using the method of the particular solution (MAPS).

\section{SINGULAR BOUNDARY METHOD}

The SBM is based on the same principle as the MFS however the SBM utilizes the same set of collocation and source points placed on the boundary $\Gamma$. The Green's function $G_{i j}$ is the fundamental solution of the Stokes equation known as Stokeslet:

$$
G_{i j}(x, y)=-\frac{1}{4 \pi}\left(\ln \left(\frac{1}{|x-y|}\right) \delta_{i j}+\frac{\left(x_{i}-y_{i}\right)\left(x_{j}-y_{j}\right)}{|x-y|^{2}}\right) .
$$

The traction fundamental solution $K_{i j}$ is

$$
K_{i j}(x, y)=-\frac{1}{\pi} \frac{\left(x_{k}-y_{k}\right)\left(x_{i}-y_{i}\right)\left(x_{j}-y_{j}\right)}{|x-y|^{4}} n_{j}(y)
$$

and corresponding pressure solution $p_{j}$ is

$$
p_{j}(x, y)=-\frac{1}{2 \pi} \frac{\left(x_{j}-y_{j}\right)}{|x-y|^{2}} .
$$

The SBM defines the solution of the homogeneous problem using the Green's functions as 


$$
\begin{aligned}
& u_{i}^{H}\left(x^{m}\right)=\sum_{k=1, k \neq m}^{n} \alpha_{k} G_{i j}\left(x^{m}, y^{k}\right)+\alpha_{m} U_{i j}\left(x^{m}, y^{m}\right), \\
& t_{i}^{H}\left(x^{m}\right)=\sum_{k=1, k \neq m}^{n} \alpha_{k} K_{i j}\left(x^{m}, y^{k}\right)+\alpha_{m} T_{i j}\left(x^{m}, y^{m}\right),
\end{aligned}
$$

where $\alpha_{k}$ are the unknown coefficients, $x^{m}$ and $y^{k}$ are the collocation and source points and $U_{i j}, T_{i j}$ are the origin intensity factors, respectively. Origin intensity factors (OIFs) are a key problem for the SBM. In order to prevent problems concerning the OIF of the Stokeslet for the 2D domains, the regularized boundary integral formulation described in Zhang et al. [13] and Sun et al. [14] has been used.

The OIFs associated with the Laplace fundamental solution for the Neumann type boundary condition can be calculated using subtracting and adding-back technique [7], [13]:

$$
Q_{i j}\left(x^{m}, y^{m}\right)=-\frac{1}{L_{m}} \sum_{k=1, k \neq m}^{n} L_{k} \frac{\partial G_{i j}\left(x^{m}, y^{k}\right)}{\partial n\left(y^{k}\right)},
$$

where $L_{j}$ is the length of the appropriate part of the boundary around point $\mathbf{x}$ (see Fig. 1). In the case of the Dirichlet boundary condition, one of the numerous possibilities of how to evaluate OIF is eqn (13), based on the get regularized boundary integral:

$$
\begin{aligned}
& U_{i j}\left(x^{m}, y^{m}\right)=-\frac{1}{L_{m}} \sum_{k=1, k \neq m}^{n} A_{k}\left[n_{i}\left(x^{m}\right) \cdot n_{j}\left(y^{k}\right) G_{i j}\left(x^{m}, y^{k}\right)-n_{i}\left(x^{m}\right)\left(y^{k}-\right.\right. \\
& \left.\left.x^{m}\right) \frac{\partial G_{i j}\left(x^{m}, y^{k}\right)}{\partial n\left(y^{k}\right)}\right] .
\end{aligned}
$$

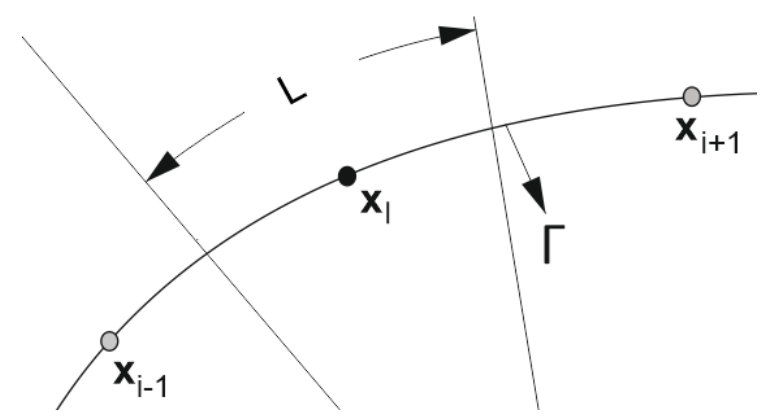

Figure 1: Part of boundary L around point $\mathbf{x}_{\mathrm{i}}$.

3.1 Dual reciprocity method: particular solution

The particular solutions $u^{\mathrm{P}}$, and $p^{\mathrm{P}}$ of eqn (6) is approximated using a linear superposition of the radial basis functions [15]:

$$
u_{i}^{\mathrm{P}}=\sum_{j=1}^{M} \beta_{j k} \bar{u}_{i k}\left(r_{j}\right), p^{\mathrm{P}}=\sum_{j=1}^{M} \beta_{j k} \bar{p} k\left(r_{j}\right),
$$

where $M$ is the number of the internal points, $\beta_{j}$ are the unknown coefficients, and $\bar{u}_{i k}$ and $\bar{p}_{k}$ are the radial basis functions which can be defined as the particular solutions of the following non-homogeneous system of eqns (15) and (16): 


$$
\begin{gathered}
\mu \frac{\partial^{2} \bar{u}_{i k}}{\partial x_{j} \partial x_{j}}-\frac{\partial \bar{p}_{k}}{\partial x_{i}}=\varphi(r) \delta_{i k} \\
\frac{\partial \bar{u}_{i k}}{\partial x_{i}}=0,
\end{gathered}
$$

where $\varphi(r)$ is the implicitly compactly supported RBF function (MQ) defined as

$$
\varphi(r)=\left(1-\frac{r}{\alpha}\right)
$$

where $r$ is Euclidean distance from the function origin and the $\alpha$ is scaling parameter used to represent the radius of circular support domain, the values outside the support domain are set as zeros. One of the main difficulties is the problem of how to find a closed-form expression of the particular solutions $\bar{u}_{i k}$ and $\bar{p}_{k}$. To solve the problem, we employ the way more detailed one described in more detail in Florez and Power [15], Bustamante et al. [16] and Happel and Brenner [17]. As the first step, an auxiliary potential $\Phi$ is defined and is used to express the velocity particular solution as follows:

$$
\bar{u}_{i k}=\frac{\partial^{2} \Phi}{\partial x_{j} \partial x_{j}} \delta_{i k}-\frac{\partial^{2} \Phi}{\partial x_{i} \partial x_{k}} .
$$

The velocity particular solution captures implicitly the continuity equation, which can be checked by supplying the above velocity field into the continuity equation. Substituting eqn (17) into the momentum eqn (15) allows us to write

$$
\mu\left(\frac{\partial^{4} \Phi}{\partial x_{j} \partial x_{j} \partial x_{k} \partial x_{k}} \delta_{i k}-\frac{\partial^{4} \Phi}{\partial x_{j} \partial x_{j} \partial x_{i} \partial x_{k}}\right)-\frac{\partial \bar{p}_{k}}{\partial x_{i}}=\varphi \delta_{i k} .
$$

The particular solution we explore is represented by the RBF approximation of the auxiliary potential used to express velocity, pressure and stress fields. The particular solution can be found splitting the momentum eqn (18) into two parts and satisfying these two equations. Then the following system is obtained:

$$
\begin{gathered}
\mu \frac{\partial^{4} \Phi}{\partial x_{j} \partial x_{j} \partial x_{k} \partial x_{k}}=\varphi(r), \\
\mu \frac{\partial^{4} \Phi}{\partial x_{j} \partial x_{j} \partial x_{i} \partial x_{k}}+\frac{\partial \bar{p}_{k}}{\partial x_{i}}=0 .
\end{gathered}
$$

The second, eqn (20), can be used to obtain pressure particular solution

$$
\bar{p}_{k}=\mu \frac{\partial^{3} \Phi}{\partial x_{j} \partial x_{j} \partial x_{k}} .
$$

The first, eqn (19), is used to compute the auxiliary potential $\Phi$ by the direct integration

$$
\begin{gathered}
\Phi(r)=\frac{r^{4}}{14400}\left(225+\frac{r}{\alpha}\left(25 \frac{r}{\alpha}-128\right)\right), r \leq \alpha \\
\Phi(r)=\frac{\alpha^{2}}{48} \ln \frac{r}{\alpha}\left(r^{2}+\frac{\alpha^{2}}{5}\right)+\frac{\alpha^{2} r^{2}}{576}+\frac{97 \alpha^{4}}{14400}, r>\alpha .
\end{gathered}
$$

Substituting the auxiliary potential $\Phi$ to eqns (17) and (21), the pressure and velocity field of a particular solution is obtained.

\subsection{System of equations}

Substituting eqns (14) and (15) into eqns (1) and (2), we have got the following $M$ equations: 


$$
\sum_{j=1}^{M} \beta_{j k} \varphi\left(r_{m j}\right)=\rho u_{k} \frac{\partial u_{i}}{\partial x_{k}}, \quad m=1,2, \ldots, M .
$$

To complete the whole system, we must add $N$ boundary conditions in all boundary nodes (see Fig. 2). These additional $N$ equations can be formulated using boundary operator $B$ as

$$
\begin{gathered}
\sum_{j=1}^{M} \beta_{j k} B\left(\bar{u}_{i k}\left(r_{m j}\right)\right)+\sum_{j=1, m \neq j}^{N} \alpha_{j m} B\left(G\left(r_{m j}\right)\right)+\alpha_{m m} B\left(U_{m}\right)=b_{0}\left(x_{m}, t\right), \\
m=1,2, \ldots, N .
\end{gathered}
$$

The system of linear equations can be written in the following form:

$$
\left[\begin{array}{cc}
\boldsymbol{K} & \boldsymbol{L} \\
\mathbf{0} & \boldsymbol{N}
\end{array}\right]\left\{\begin{array}{l}
\alpha \\
\beta
\end{array}\right\}=\left\{\begin{array}{c}
\mathbf{b}_{\mathbf{0}} \\
\mathbf{S}
\end{array}\right\}
$$

where

$$
K_{i j}=B\left(G\left(r_{i j}\right)\right), \quad L_{i j}=B\left(\bar{u}_{i k}\left(r_{m j}\right)\right)
$$

and

$$
N_{i j}=\varphi\left(r_{m j}\right), \quad S_{i}=\rho u_{k} \frac{\partial u_{i}}{\partial x_{k}} .
$$

The computational algorithm deals with the nonlinearity caused by transport terms adopting Pickard iterative scheme together with the variation of right-hand side evaluated using transport terms $S_{i}$ (eqn (27)). The part of the equation system eqn (25) used to construct a particular solution will be sparse and the system will be computationally more efficient. The other effect is that the local nature of kernel functions used to create particular solution enables the solution of problems with higher Reynolds number.

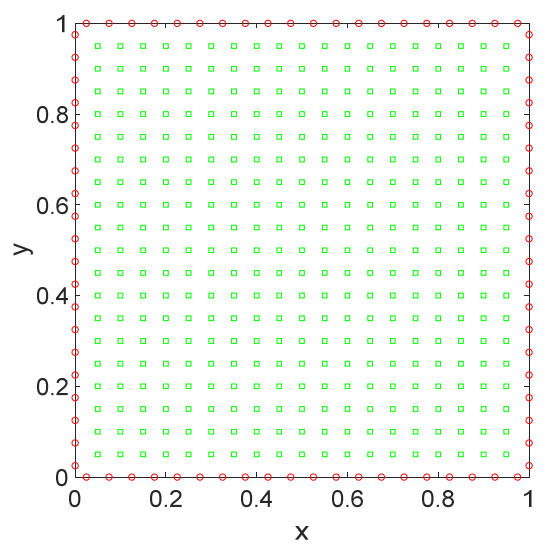

Figure 2: The boundary and internal nodes distribution covering the global computational domain used for SBMDR method.

\section{SIMULATION OF THE LID-DRIVEN CAVITY PROBLEM}

Lid-driven cavity flow is being used as a standard test case for the validation of numerical solutions of incompressible Navier-Stokes flow. The upper wall of the cavity motions with a velocity $u=1$, and no-slip impermeable boundary conditions are considered along the other 
three walls. The geometry and velocity boundary conditions are demonstrated in Fig. 3. The dilemma of this problem remains in the presence of the singularities of the pressure and velocity at the two top corners of the cavity. Therefore, the amount of the computational nodes utilized decreases towards the corners of the cavity (see Fig. 3) to catch the high pressure and velocity function gradients.

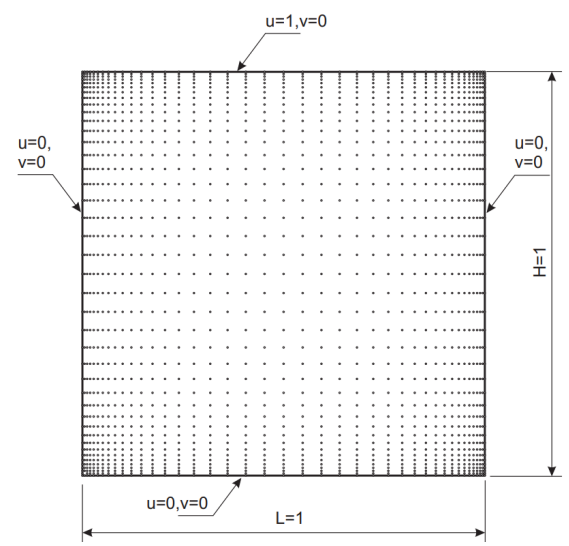

Figure 3: The lid-driven cavity problem geometry, boundary conditions, and distribution of internal nodes.

The steady solution using the SBMDR is achieved when the discrepancy between two consecutive time steps (in the definition of the infinity norm) is lower than specified, $\varepsilon_{t o l}=1 \times 10^{-6}$ is applied for presented numerical computations. The results of the SBMDR are confronted with the solution represented in AbdelMigid et al. [18] for the Reynolds number $\operatorname{Re}=3200$; the vertical velocity components along a horizontal line and the horizontal velocity components along a vertical line, straight to the center of the cavity, are plotted in Fig. 4. Fig. 5 shows streamlines and pressure contours for Reynolds number of $\operatorname{Re}=3200$.

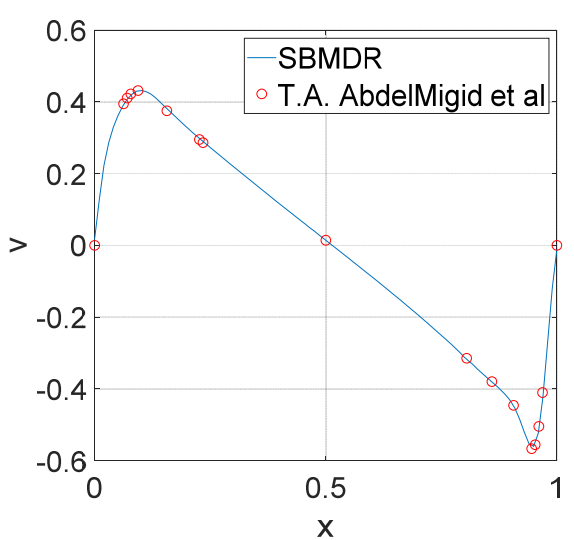

(a)

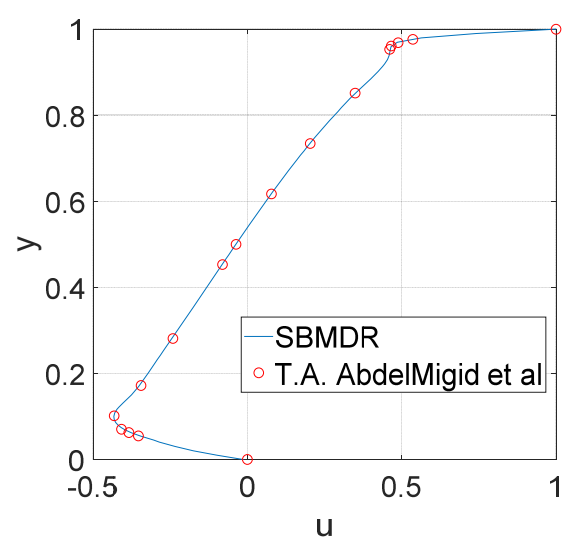

(b)

Figure 4: Lid-driven cavity, $\operatorname{Re}=3200$. (a) Vertical velocity components along horizontal line $y=0.5$; and (b) Horizontal velocity components along vertical line $x=0.5$. 

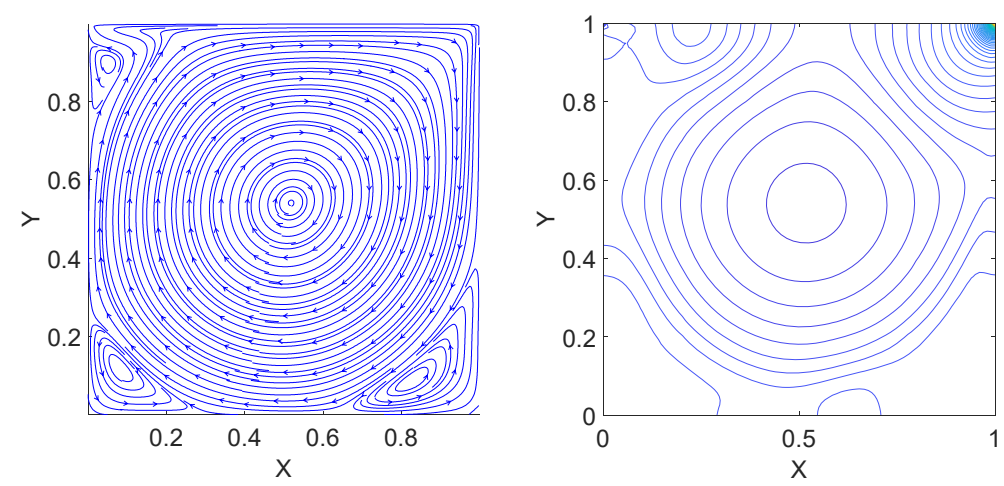

Figure 5: Streamlines and pressure contours for lid-driven cavity problem, $\mathrm{Re}=3200$.

\section{SIMULATION OF THE BACKWARD-FACING STEP FLOW}

The flow over a backward-facing step is extensively tested arrangement of the fluid flow in a channel. A couple of the numerical and experimental studies of the $2 \mathrm{D}$ steady incompressible flows have been disclosed (e.g. Erturk [19]). The inlet velocity is considered to be horizontal with a parabolic distribution, and maximum inflow velocity is $u_{\max }=1.5$, and the average inflow velocity is $u_{a}=1$. The Reynolds number could be defined as

$$
R e=\frac{u_{a} H}{v},
$$

where $H$ is the height of the channel (see Fig. 6). The geometry configuration and boundary conditions are shown in Fig. 6.

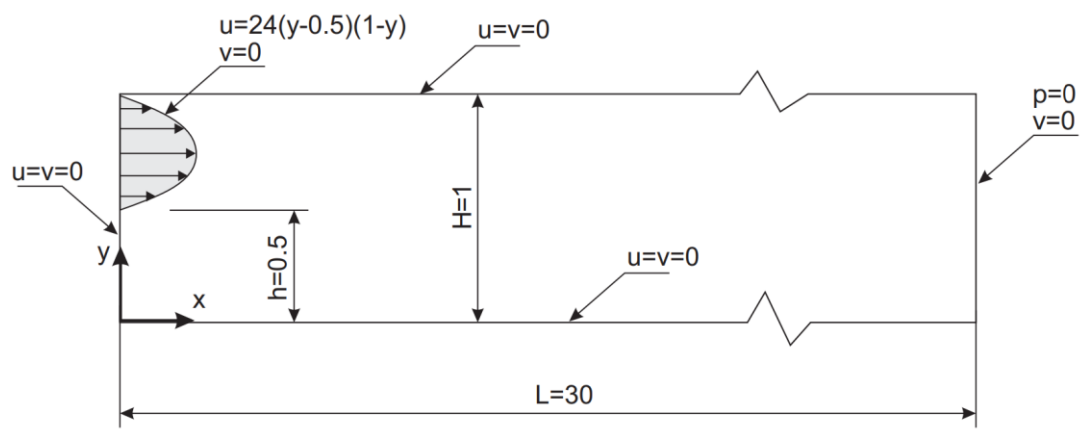

Figure 6: The geometry of the backward-facing step problem.

The entire domain is covered by a uniform distribution of computational nodes, 261 nodes in the $x$-direction and 31 in the $y$-direction. The primary values of all quantities are set to zero. The steady solution is again attained when the tolerance between two consecutive time steps is lower than a designated value.

Fig. 7 demonstrates streamlines and pressure contours for $\operatorname{Re}=800$, and Fig. 8 demonstrates the horizontal velocity components in vertical profiles. Comparing the results of the SBMDR for $\mathrm{Re}=800$ with results of Erturk [19] show the close agreement of the proposed formulation. 


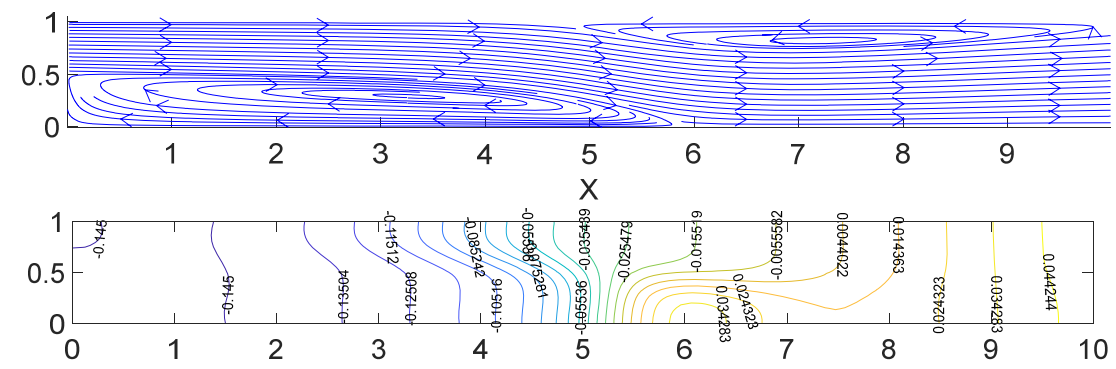

Figure 7: Stream function contours (streamlines) and pressure contours for the backward-facing step, $\mathrm{Re}=800$.
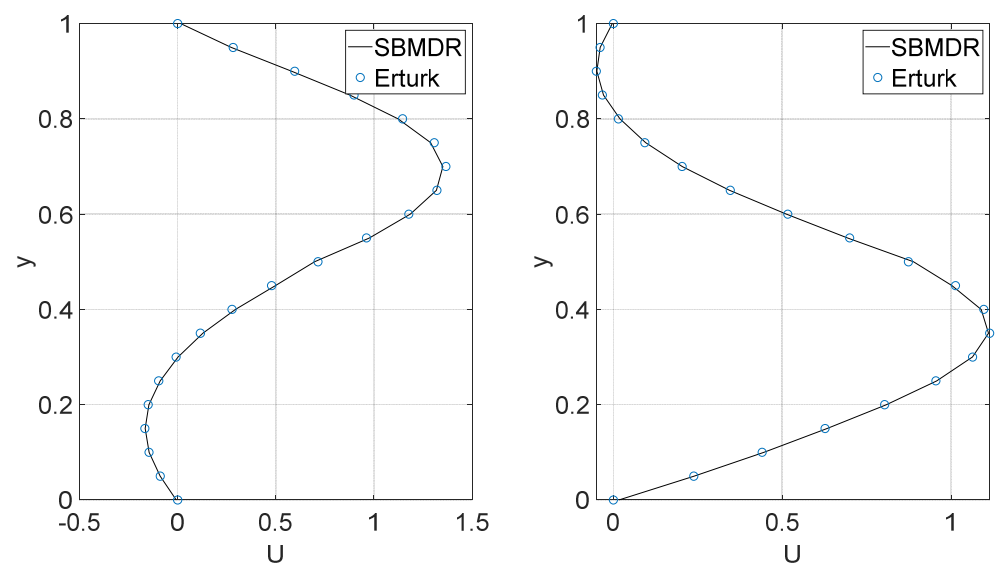

Figure 8: Backward-facing step, the horizontal velocities at $\mathrm{x}=3$ and $\mathrm{x}=7$ profiles for $\operatorname{Re}=800$.

\section{CONCLUSIONS}

This paper documents the experiment to apply the global SBM and DRM (SBMDR) for the solution the incompressible laminar flow outlined by a primitive variable form of Navier-Stokes equation. Two types of benchmarking problems, lid-driven cavity and backward-facing step, have been successfully tested. This type of boundary collocation method seems to be quite a useful alternative to the solutions of incompressible fluid flow using the boundary based numerical schemes. The dual reciprocity technique implemented using implicitly compactly supported RBFs generates sparse characteristic matrix and enables the method to obtain reasonable results even for high Reynolds number. The presented numerical code and numerical results need to be more analyzed in the sense of precision, convergence and computational demands.

\section{ACKNOWLEDGEMENTS}

This contribution is the result of the project funded by the Scientific Grant Agency of Slovak Republic (VEGA) No. 1-0716-17. Internal grant UNIZA "Vyhodnotenie statickej penetračnej skúšky použitím neurónových sietí”. 


\section{REFERENCES}

[1] Gingold, R.A. \& Monaghan, J.J., Smoothed particle hydrodynamics: Theory and applications to non-spherical stars. Monthly Notices of the Royal Astronomical Society, 181, pp. 375-389, 1977.

[2] Kansa, E.J., Multiquadrics - a scattered data approximation scheme with application to computational fluid dynamics. Computers \& Mathematics with Applications, 19, pp. 127-145, 1990.

[3] Sanyasiraju, Y. \& Chandhini, G., Local radial basis function based grid-free scheme for unsteady incompressible viscous flows. Journal of Computational Physics, 227, pp. 8922-8948, 2008.

[4] Shu, C., Ding, H. \& Yeo, K.S., Local radial basis function-based differential quadrature method and its application to solve two-dimensional incompressible Navier-Stokes equations. Computer Methods in Applied Mechanics and Engineering, 192, pp. 941-954, 2003.

[5] Atluri, S.N., Kim, H.-G. \& Cho, J.Y., A critical assessment of the truly Meshless Local Petrov-Galerkin (MLPG), and Local Boundary Integral Equation (LBIE) methods. Computational Mechanics, 24, 348-372, 1999.

[6] Lin, H. \& Atluri, S.N., The Meshless Local Petrov-Galerkin (MLPG) method for solving incompressible Navier-Stokes equations. Computer Modeling in Engineering \& Sciences, 2, pp. 117-142, 2001.

[7] Zhu, T., Zhang, J. \& Atluri, S., A meshless numerical method based on the local boundary integral equation (LBIE) to solve linear and nonlinear boundary value problems. Engineering Analysis with Boundary Elements, 23, pp. 375-389, 1999.

[8] Sladek, V., Sladek, J., Atluri, S.N. \& Van Keer, R., Numerical integration of singularities in meshless implementation of local boundary integral equations. Computational Mechanics, 25, pp. 394-403, 2000.

[9] Kováŕík, K. \& Mužík, J., A meshless solution for two dimensional density-driven groundwater flow. Engineering Analysis with Boundary Elements, 37, pp. 187-196, 2013.

[10] Bui, T.T. \& Popov, V., Radial basis integral equation method for Navier-Stokes equations. Boundary Elements and Other Mesh Reduction Methods, vol. 49, WIT Press, pp. 141-150.

[11] Popov, V. \& Thanh Bui, T., A meshless solution to two-dimensional convectiondiffusion problems. Engineering Analysis with Boundary Elements, 34, pp. 680-689, 2010.

[12] Kováŕík, K., Mužík, J. \& Mahmood, M.S., A meshless solution of two dimensional unsteady flow. Engineering Analysis with Boundary Elements, 36, pp. 738-743, 2012.

[13] Zhang, Y.M., Sun, F.L., Young, D.L., Chen, W. \& Gu, Y., Average source boundary node method for potential problems. Engineering Analysis with Boundary Elements, 70, pp. 114-125, 2016.

[14] Sun, F.L., Zhang, Y.M., Young, D.L. \& Chen, W., A new boundary meshfree method for potential problems. Advances in Engineering Software, 100, pp. 32-42, 2016.

[15] Florez, W.F. \& Power, H., Comparison between continuous and discontinuous boundary elements in the multidomain dual reciprocity method for the solution of the two-dimensional Navier-Stokes equations. Engineering Analysis with Boundary Elements, 25, pp. 57-69, 2001.

[16] Bustamante, C.A., Florez, W.F. \& Power, H., A global meshless collocation particular solution method for solving the two-dimensional NavierStokes system of equations. Computers and Mathematics with Applications, 65, pp. 1939-1955, 2013. 
[17] Happel, J. \& Brenner, H., Low Reynolds Number Hydrodynamics, 2nd ed., Martinus Nijhoff Publishers: The Hague, 1983.

[18] AbdelMigid, T.A., Saqr, K.M., Kotb, M.A. \& Aboelfarag, A.A., Revisiting the liddriven cavity flow problem: Review and new steady state benchmarking results using GPU accelerated code. Alexandria Engineering Journal, 56(1), pp. 123-135, 2017.

[19] Erturk, E., Numerical solutions of 2-D steady incompressible flow over a backwardfacing step, Part I: high Reynolds number solutions. Computers \& Fluids, 37, pp. 633$55,2008$. 